\title{
Metal-Semiconductor Transition in Armchair Carbon Nanotubes by Symmetry Breaking
}

\author{
Yan Li, Slava V. Rotkin $*$ and Umberto Ravaioli \\ Beckman Institute for Advanced Science and Technology, \\ University of Illinois at Urbana Champaign, 405 N. Mathews, Urbana, Illinois 61801
}

(Dated: June 29, 2021)

\begin{abstract}
The electronic band structure of armchair carbon nanotubes may be considerably modified by potentials with angular dependence. Different angular modes $V_{q} \sim \cos q \theta$ have been studied within a tight-binding scheme. Using symmetry arguments, we demonstrate a band gap opening in these metallic nanotubes when certain selection rules are satisfied for both potential and nanotube structure. We estimate the band gap opening as a function of both the external potential strength and the nanotube radius and suggest an effective mechanism of metal-semiconductor transition by combination of different forms of perturbations.
\end{abstract}

Armchair single-wall carbon nanotubes (SWNTs) with indices $(n, n)$ are metallic with two subbands crossing at the Fermi level, which is allowed by opposite parities of these two subbands with regard to the $n$ vertical mirror reflections $\sigma_{v}$. It is interesting to explore if one can break the mirror symmetry and generate a band gap by choosing appropriate angular perturbations, so that one can modify and control nanotube material properties for applications. It was recently proposed to use a very inhomogeneous electric field to induce metal-semiconductor transition (MST) in armchair SWNTs [1]. Other types of circumferential perturbations were studied including intra-rope interaction [2], twisting or bending [3], squashing [4, 5, 6, 7] and applying uniform perpendicular electric fields $[8,9]$. Some perturbations indeed were found to induce MST, which was attributed partially to the breaking of SWNT mirror symmetry. However, there still remains an open question if mirror symmetry breaking (MSB) is a sufficient condition to bring in a band gap in armchair tubes. For example, in the case of bending or applying a uniform electric field, by rotating the armchair nanotube, one can always find an alignment that breaks all vertical mirror symmetries but the nanotube remains metallic, which cannot be explained by the MSB argument alone.

In this letter, we apply potentials with angular dependence to modulate the band structure and electronic properties of the armchair SWNTs. We choose angular modes $V_{q}=V_{0} \cos q \theta$ or their combinations to study gap opening within an orthogonal $\pi$-orbital tight-binding (TB) scheme. We found that (1) breaking symmetries about all the vertical mirror reflections and $C_{2}$ rotations (see below) is required to mix the two linear subbands near the Fermi level; (2) selection rules of subband coupling impose additional requirement on the angular momentum of the perturbation, e.g. a single mode with odd $q$ does not generate any band gap; (3) for modes with even $q$, MST is only possible for tubes with specific indices $n$ to satisfy selection rules described below.

Selection Rules. The symmetry group of an $(n, n)$ armchair SWNTs consists of vertical mirror planes $\sigma_{v}$, horizontal mirror planes $\sigma_{h}$ and rotation axes $C_{2} \equiv$ $\left\{U, U^{\prime}\right\}$ (Fig. 1) . Every electron state can be labeled by a set of quantum numbers: an axial wave number $k$, an angular quantum number $m$, a parity with regard to the vertical mirror reflection $\sigma_{v}(A / B)$ and a parity to the horizontal mirror reflection $\sigma_{h}(+/-)$. There are also two sets of $C_{2}$ axes $U$ and $U^{\prime}$, which are perpendicular to the tube axis. The two linear subbands $|\pi\rangle$ and $\left|\pi^{*}\right\rangle$, both have $m=n$ and even parities about $\sigma_{h}$, while their parities about $U, U^{\prime}$ and $\sigma_{v}$ are opposite [10]. It is the different parities that allow the two subbands to cross and be degenerate at the Fermi points. We do not consider potentials with an axial space dependence, thus, the quantum number $k$ is conserved.

What are the symmetry conditions required for MST in armchair nanotube?

Rule 1 All symmetries about vertical mirror planes $\sigma_{v}$ and $C_{2}$ axes $U, U^{\prime}$ must be broken simultaneously.

The selection rules of subband coupling impose additional requirement on the angular quantum number of the potential as well as the nanotube indices. Assume

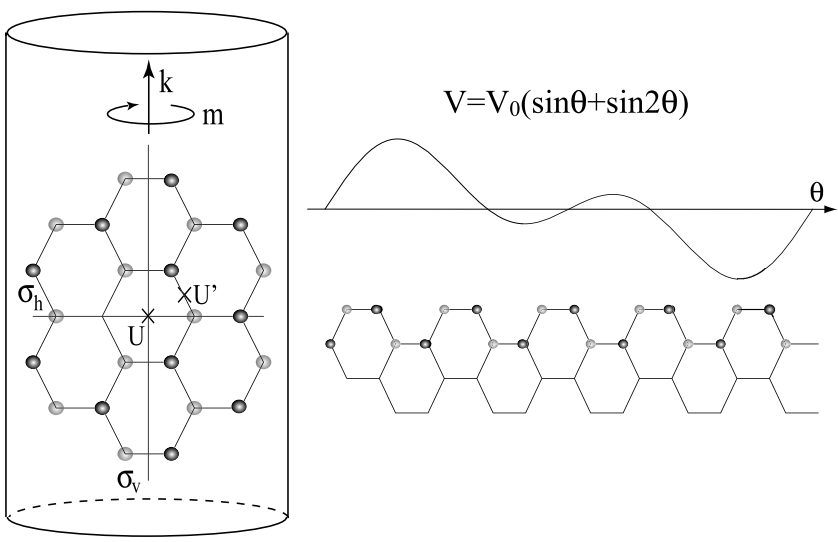

FIG. 1: Left: Symmetry operations of an $(n, n)$ armchair nanotube. Right: Unwrapped unit cell of a $(5,5)$ nanotube and schematics of an external potential of the form $V=V_{0}(\sin \theta+\sin 2 \theta)$. 
that the external potential has only one angular mode $V_{q}(\theta) \sim \cos q\left(\theta+\theta_{0}\right)$, where an arbitrary offset $\theta_{0}$ is zero when a mirror plane of the potential coincides with the one of the SWNT. The conservation of total quantum numbers $k$ and $m$ imposes the selection rules for direct subband coupling in an $(n, n)$ SWNT: $\delta k=0$ and $\delta m= \pm q+2 n j$, with $j$ an integer. The indirect interaction between $|\pi\rangle$ and $\left|\pi^{*}\right\rangle$ states $H_{\pi \pi^{*}}$ can thus be represented by a Feynman-like diagram, or as a perturbation series of the coupling order $\mu$ (Fig. 2(a)). Here all allowed intermediate states $\left\{m_{i}, s_{i}\right\}$ are states of the given angular momentum $m$ and pseudo-spin $s$, with $s= \pm 1$ denoting the conduction and valence bands respectively. Due to the symmetry of electron and hole bands, contributions of even $\mu$ cancel out as shown in Fig. 2(b) (a detailed example will be shown below). Thus, only for odd coupling order $\mu$, there can be a non-vanishing coupling between $|\pi\rangle$ and $\left|\pi^{*}\right\rangle$ states. The most important contribution comes from the lowest possible $\mu$ which is $\mu_{0}=2 n / \operatorname{gcd}(2 n, q)$, where gcd is the greatest common divisor. We formulate the second rule for the MST in the single mode angular potential $V_{q}$ :

\section{Rule 2}

$$
\mu_{0} \equiv \frac{2 n}{\operatorname{gcd}(2 n, q)}=\text { odd }
$$

One conclusion we can draw immediately is that there is no MST when $q$ is odd, because then $\mu_{0}$ is even, which violates rule 2 . This is consistent with the absence of the band gap for armchair SWNTs under a uniform perpendicular electric field, i.e. $q= \pm 1$ [9].

By nearly degenerate perturbation theory, we derive the dependence of the band gap opening on the angular offset $\theta_{0}$. We already know that when $\theta_{0}=0$, there shall be no band gap according to rule 1. By changing the relative alignment of the SWNT and the potential, a band gap starts to develop and $E_{g}$ can be estimated as:

$$
E_{g}\left(\theta_{0}\right)=E_{g}^{\max } \sin \left(\mu_{0} q \theta_{0}\right) .
$$

It is self-evident that the maximum of the band gap is for $\theta_{0}=\pi /\left(2 \mu_{0} q\right)$ when $V_{q}$ is so aligned that the potential is antisymmetric about one of the vertical mirror planes.

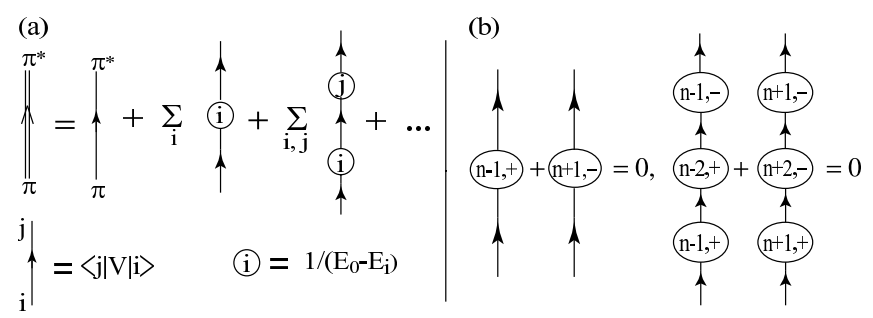

FIG. 2: (a) Feynman diagram for interaction between $|\pi\rangle$ and $\left|\pi^{*}\right\rangle$ states. $|i\rangle=\left|m_{i}, s_{i}\right\rangle$. (b)Two examples of the contributions that cancel out for $\mu=$ even.
The case with $q=2$ is reminiscent of squashing an armchair SWNT. Our analysis explains why a small band gap was found in a $(5,5)$ SWNT in Ref. [4 but not in $(6,6)$ SWNT in Ref. [6] or $(8,8)$ SWNT in Ref. [7]. Since $\mu_{0}=n$ for $q=2$, only SWNTs with odd $n$ can open a band gap. In $(8,8)$ and $(6,6)$ armchair nanotubes there should be no mixing between the crossing subbands. For odd $n$, the band gap opening is roughly $E_{g} \propto V_{0}^{n} \sin \left(2 n \theta_{0}\right)$ and decreasing for large radius nanotubes due to the higher order of perturbation. The maximum band gap of a $(5,5)$ SWNT by the $q=2$ potential is of the order of $0.1 \mathrm{eV}$ within our TB calculation.

The most interesting case for potential applications is that the potential has short oscillation period: $q=2 n$, which yields direct mixing to the coupling order $\mu_{0}=1$. Assuming no overlap between orbitals on different atomic sites, an analytical expression for the band gap can be obtained by the degenerate perturbation theory:

$$
E_{g}=\sqrt{3} V_{0} \sin \left(2 n \theta_{0}\right) .
$$

Since $|\pi\rangle$ and $\left|\pi^{*}\right\rangle$ are now directly coupled, the band gap is proportional to the perturbation and the relation in Eq. (2) holds up to a few eV. Potential of this form $(q=2 n)$ requires changing the sign of the electrostatic potential alternatively on neighboring carbon atoms. One can possibly generate such perturbations by twisting, chemical/biological decoration of the tube or by using high multipoles of very inhomogeneous potential.

The potential with mixed Fourier components. Realistic perturbations usually have more than one dominating angular mode, which makes analytical expressions tedious. On the other hand, the interplay of the different angular components may result in stronger and more interesting perturbation of the electronic properties of the SWNTs. For example, the transport behavior of a deformed nanotube may change under the control of the gate potential 11]. When the strength of the field across the SWNT is large, the gate potential shifts the Fermi level and also modifies the band structure. In the simplest case, only two angular components are present: $H_{1}=V_{1} \cos \left(\theta+\theta_{1}\right)+V_{2} \cos \left(2\left(\theta+\theta_{2}\right)\right)$. Here $\theta_{1}$ and $\theta_{2}$ are defined as the angular offsets of the mirror planes of $V_{1}$ and $V_{2}$ with regard to that of the SWNT.

The coupling order $\mu_{0}$ is now a function of both $q_{1}$ and $q_{2}$. For $q_{1}=1, q_{2}=2$, the lowest allowed coupling is of the 3rd order through $|\pi\rangle \leftrightarrow\left|m_{1}, s_{1}\right\rangle \leftrightarrow\left|m_{2}, s_{2}\right\rangle \leftrightarrow$ $\left|\pi^{*}\right\rangle$ and there are 24 possible sets of intermediate states $\left\{m_{1}, s_{1} ; m_{2}, s_{2}\right\}$ :

$$
\left(m_{1}, m_{2}\right)=\left\{\begin{array}{l}
( \pm 1, \mp 1) \\
( \pm 1, \pm 2) \\
( \pm 2, \pm 1)
\end{array} \otimes\left(s_{1}, s_{2}\right)=\left\{\begin{array}{c}
( \pm 1, \pm 1) \\
( \pm 1, \mp 1)
\end{array} .\right.\right.
$$

Here $m_{1,2}= \pm 1, \pm 2$ are the quantum numbers relative to $m=n$. In general case, the band gap opening is related to the off-diagonal element $H_{\pi \pi^{*}}$ in the $2 \times 2$ matrix of 
$\pi$ and $\pi^{*}$ states, which can be written via contributions from different intermediate sets of $|i\rangle \equiv\left|m_{i}, s_{i}\right\rangle$ :

$$
\begin{aligned}
H_{\pi \pi^{*}}^{(\mu)}(\{i\}) & =\frac{\left\langle\pi\left|H_{1}\right| 1\right\rangle \ldots\left\langle i\left|H_{1}\right| i+1\right\rangle \ldots\left\langle\mu-1\left|H_{1}\right| \pi^{*}\right\rangle}{\left(E_{0}-E(1)\right) \ldots\left(E_{0}-E(\mu-1)\right)} \\
H_{\pi \pi^{*}} & =\sum_{\left\{m_{i}, s_{i}\right\}} H_{\pi \pi^{*}}^{(\mu)}(\{i\}),
\end{aligned}
$$

where $E_{0}=0$ for symmetric $\pi$ and $\pi^{*}$ bands and $E(i)$ is the eigen energy of the electron state $|i\rangle$.

The matrix element $\langle V\rangle=\left\langle i\left|V_{q} \cos q\left(\theta+\theta_{q}\right)\right| i+1\right\rangle$ can be simplified by neglecting the coupling between sublattices in an envelope function approximation:

$$
\begin{aligned}
\langle V\rangle & \approx \delta_{\Delta m, \pm q} \frac{V_{q}}{4} e^{i \Delta m \theta_{q}}\left(1+s_{i} s_{i+1} e^{i\left(\phi_{m_{i+1}}-\phi_{m_{i}}\right)}\right), \\
e^{i \phi_{m}(k)} & =C_{m}^{A}(k) / C_{m}^{B}(k),
\end{aligned}
$$

where $\Delta m=m_{i}-m_{i+1}$ and $C_{m}^{A, B}(k)$ are the coefficients of the Bloch components on A and B sub-lattices in the electronic wave functions. Since one is mostly interested in the properties of electronic states near the Fermi level, nearly degenerate perturbation theory will be appropriate as long as the perturbation is small $\delta=V_{0} / E_{10} \ll 1$, where $E_{10}=v_{R} / R$ is the characteristic energy spacing between subbands. Substituting the allowed transitions of Eq. (3) into Eq. (4) and summing up, we can get the analytical expression for the band gap at $k=k_{F}$ :

$$
\begin{aligned}
E_{g} & \approx 2\left|H_{\pi \pi^{*}}^{(3)}\right|=\frac{V_{1}^{2} V_{2}}{2 E_{10}^{2}} \sin \left(2 \Delta \theta_{12}\right)\left(\sin \frac{\pi}{3 n}+\sin \frac{\pi}{6 n}\right) \\
& \approx \frac{\pi}{4 n} E_{10}\left(\frac{V_{1}^{2} V_{2}}{E_{10}^{3}}\right) \sin \left(2 \Delta \theta_{12}\right) \sim \delta^{3} R^{-2}
\end{aligned}
$$

where $\Delta \theta_{12}=\theta_{2}-\theta_{1}$ and the dimensionless potential $\delta$ is defined as $\left(V_{1}^{2} V_{2}\right)^{1 / 3} / E_{10}$. The $R^{-2}$ dependence makes

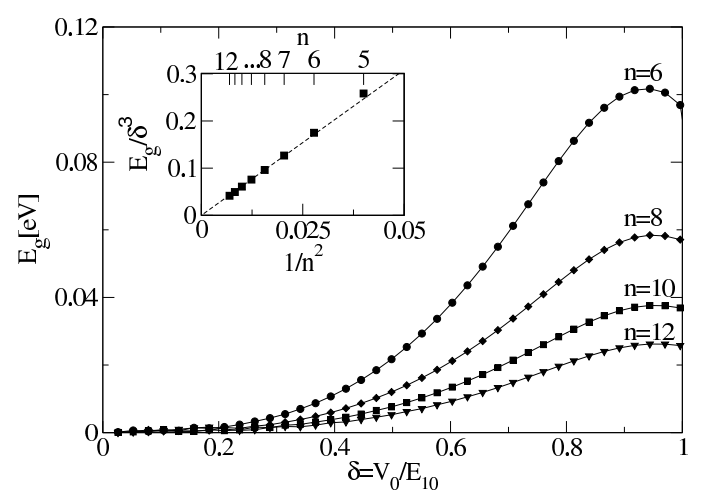

FIG. 3: The band gap opening as a function of the dimensionless potential $\delta=V_{0} / E_{10}$ for $(n, n)$ SWNTs with $n=6,8,10,12$, and $V_{1}=V_{2} \equiv V_{0}$. Inset: The ratio $E_{g} / \delta^{3}$ vs. $n^{-2}$ for TB results (solid squares) and results of perturbation theory (dashed line) with $n=5, \ldots, 12$.
$E_{g}$ of the order of a secondary band gap in quasi-metallic SWNTs, which decays with the inverse square of the radius. One should notice that $E_{g}$ is only related to the relative angle $\Delta \theta_{12}$. When $\Delta \theta_{12}=0$, the mirror planes of the two components coincide and perturbation theory predicts no band gap whether or not the total potential breaks the mirror symmetry. The maximum band gap happens at $\Delta \theta_{12}= \pm \pi / 4$, which corresponds to the configuration that the nodes of the two potential components overlap, as confirmed by numerical TB results when $n \geq 5$ (Fig. 3). Since this combination $\left(q_{1}=1\right.$ and $q_{2}=2$ ) always gives a secondary band gap unless $\Delta \theta_{12}=0$, which can be tens or hundreds of $\mathrm{meV}$ for SWNTs with a moderate radius, it may be an effective mechanism to induce MST in armchair nanotubes and put them into tunable metallic field-effect transistors [1].

In conclusion, we derived the selection rules for the metal-semiconductor transition of armchair SWNTs under an external circumferential perturbation within the orthogonal $\pi$-orbital TB scheme. We evaluated the band gap opening as a function of the external potential strength and its angular alignment with SWNT mirror planes. Combinations of perturbations of different angular modes are shown to open the gap up to $0.1 \mathrm{eV}$ for $(6,6)$ SWNT and may represent an effective mechanism of the metal-semiconductor transition.

This work was supported by the ARMY DURINT contract SIT 527826/222708 and NSF grant ITR/SY 0121616. S.V.R acknowledges support through DoE grant DE-FG02-01ER45932, NSF grant ECS 04-03489 and EEC-0228390.

* Present address: Department of Physics, Lehigh University, 16 Memorial Drive East, Bethlehem PA 18015, USA.

[1] S. V. Rotkin and K. Hess, Appl. Phys. Lett 84, 3139 (2004).

[2] P. Delaney, H. J. Choi, J. Ihm, S. G. Louie, and M. L. Cohen, Nature 391, 466 (1998).

[3] C. L. Kane and E. J. Mele, Phys. Rev. Lett. 78, 1932 (1997).

[4] C.-J. Park, Y.-H. Kim, and K. J. Chang, Phys. Rev. B 60, 10656 (1999).

[5] P. E. Lammert, P. Zhang, and V. H. Crespi, Phys. Rev. Lett. 84, 2453 (2000).

[6] O. Gülseren, T. Yildirim, S. Ciraci, and Ç. Kılıç, Phys. Rev. B 65, 155410 (2002).

[7] J.-Q. Lu, J. Wu, W. Duan, F. Liu, B.-F. Zhu, and B.-L. Gu, Phys. Rev. Lett. 90, 156601 (2003).

[8] J. O'Keeffe, C. Wei, and K. Cho, Appl. Phys. Lett 80, 676 (2002).

[9] Y. Li, S. V. Rotkin, and U. Ravaioli, Nano Letters 3, 183 (2003).

[10] T. Vuković, I. Milošević, and M. Damnjanović, Phys. Rev. B 65, 045418 (2002).

[11] M. J. Biercuk, N. Mason, , J. M. Chow, and C. M. Marcus, cond-mat/0312276 (2003). 